Bull. Korean Math. Soc. 52 (2015), No. 4, pp. 1107-1112

http://dx.doi.org/10.4134/BKMS.2015.52.4.1107

\title{
THE EXTENDED REAL LINE AS A JULIA SET
}

\author{
Monireh Akbari and Maryam Rabil
}

Abstract. A recursive family $\left\{F_{n}\right\}$ of holomorphic functions on the Riemann sphere is defined and some elementary properties of this family is described. Then the Julia set of $F_{n}$ is computed. Finally this family as a real recursive family is studied and it is shown that $F_{n}$ is chaotic on a specific subset of $\mathbb{R}$.

\section{Introduction and preliminaries}

Suppose $\hat{\mathbb{C}}$ is the Riemann sphere and $f: \hat{\mathbb{C}} \rightarrow \hat{\mathbb{C}}$ is a non-constant holomorphic map. By $f^{n}$ we mean $\underbrace{f \circ \cdots \circ f}_{n \text { times }}$. The point $p \in \hat{\mathbb{C}}$ is an attracting (repelling) fixed point of $f$, if $f(p)=p$ and $\left|f^{\prime}(p)\right|<1\left(\left|f^{\prime}(p)\right|>1\right)$. The basin of attraction of $p$ is the set of all points $z$ such that $\lim _{n \rightarrow \infty} f^{n}(z)=p$. Generally, if $f^{k}(p)=p$ for some $k \geq 1$, then $p$ is called a periodic point of $f$.

The Fatou set of $f$ consists of all points $z \in \hat{\mathbb{C}}$ that have an open neighborhood on which $\left\{f^{n}\right\}$ forms a normal family. The Julia set of $f$, denoted by $J(f)$, is the complement of the Fatou set. It is clear from the definition that the Fatou set is an open subset of $\hat{\mathbb{C}}$ and therefore the Julia set is a closed subset of $\hat{\mathbb{C}}$. The attracting fixed points belong to the Fatou set and the repelling fixed points belong to the Julia set and also the boundary of the basin of attraction of a fixed point is a subset of the Julia set.

Holomorphic functions on the Riemann sphere have various Julia sets with very different shapes. Among these different sets, circles, the extended real line and closed intervals are well-known smooth Julia sets. More specifically the following results are known.

- The Julia set of a polynomial of degree $d \geq 2$ is homeomorphic to a closed interval if and only if it is linearly conjugate to $\pm \phi_{d}$, where $\phi_{d}$ is the Chebyshev polynomial of degree $d$ (see [3, Lemma B.3]).

Received December 25, 2013.

2010 Mathematics Subject Classification. 37F50.

Key words and phrases. Julia set, recursive relation, conjugacy, rational function. 
- If the Julia set of a rational function $f(z)$ of degree $d \geq 2$ is the unit circle, then

$$
f(z)=\alpha \frac{z-a_{1}}{1-\overline{a_{1}} z} \frac{z-a_{2}}{1-\overline{a_{2}} z} \cdots \frac{z-a_{d}}{1-\overline{a_{d}} z}
$$

where $a_{j} \in \hat{\mathbb{C}} \backslash \partial \mathbb{D}, \alpha \in \partial \mathbb{D}$, and $\mathbb{D}$ is the unit disc (see [4, Lemma 15.5]).

- The Julia set of

$$
f(z)=\alpha z \frac{z-a_{1}}{1-\overline{a_{1}} z} \frac{z-a_{2}}{1-\overline{a_{2}} z} \cdots \frac{z-a_{d}}{1-\overline{a_{d}} z}
$$

where $a_{j} \in \mathbb{D}, \alpha \in \partial \mathbb{D}$, and $d \geq 1$, is the unit circle (see [4, page 73]).

- If $f$ is a polynomial of degree $d \geq 2$, then its Julia set is the unit circle if and only if $f(z)=a z^{d}$, where $a \in \partial \mathbb{D}$ (see [1, Theorem 1.3.1]).

It is also known that if $f$ and $g$ are two commuting $(f \circ g=g \circ f)$ rational functions of degree at least two, then $J(f)=J(g)$ (see [1, Theorem 4.2.9]). Another basic result states that if $f$ and $g$ are two rational functions that are conjugate under a Möbius function $\varphi$ (i.e., $f \circ \varphi=\varphi \circ g$ ), then the image of the Julia set of $g$ under $\varphi$ is the Julia set of $f$ (see [1, Theorem 3.1.4]). Therefore under suitable conjugacy, some of the above statements about the unit circle can be modified to $\mathbb{R} \cup\{\infty\}$.

Recall that a continuous function $f: X \rightarrow X$, where $X$ is a metric space with metric $d$, is called chaotic if the following three conditions hold:

1. The set of the periodic points of $f$ is dense in $X$.

2. $f$ is topologically transitive (i.e., for every pair of open subsets $U, V$ of $X$ there exists $k>0$ such that $\left.f^{k}(U) \cap V \neq \emptyset\right)$.

3. $f$ has sensitive dependence on initial conditions (i.e., there exists $\delta>0$ such that for any $x \in X$ and any open subset $U$ containing $x$, there exist $y \in U$ and $n \geq 0$ such that $\left.d\left(f^{n}(x), f^{n}(y)\right)>\delta\right)$.

See $[2$, page 50$]$ for more details.

In this paper we introduce a family $\left\{F_{n}\right\}$ of rational functions recursively and show that the Julia set of $F_{n}$ is $\mathbb{R} \cup\{\infty\}$. We use two different methods to compute the Julia set of $F_{n}$, firstly by exploiting the commutativity of this family and secondly by establishing the conjugacy of this family with another well known family of functions. Finally, we study $F_{n}$ as a real function and show that it is chaotic on a specific subset of $\mathbb{R}$.

\section{The recursive family and its properties}

In this section we will define a family of rational functions $\left\{F_{n}\right\}$ on $\hat{\mathbb{C}}$ recursively and determine their Julia sets.

Recall that the Newton iteration function associated to $f(z)=z^{2}+1$, that is $N(z)=z-\frac{f(z)}{f^{\prime}(z)}=\frac{z^{2}-1}{2 z}$, satisfies the relation

$$
F_{2}(z)=\frac{z F_{1}(z)-1}{z+F_{1}(z)}
$$


for $F_{1}(z)=z$ and $F_{2}(z)=N(z)$. Motivated by this observation we define the following recursive family.

Let $F_{1}(z)=z$ and, for $n \geq 2$,

$$
F_{n}(z)=\frac{z F_{n-1}(z)-1}{z+F_{n-1}(z)}
$$

Note that if $F_{n-1}\left(z_{0}\right)=\infty$, then

$$
F_{n}\left(z_{0}\right)=\lim _{z \rightarrow z_{0}} \frac{z F_{n-1}(z)-1}{z+F_{n-1}(z)} .
$$

The following lemmas describe some properties of this family.

Lemma 2.1. For $k \geq 1$ and $n>k$,

$$
F_{n}(z)=\frac{F_{k}(z) F_{n-k}(z)-1}{F_{k}(z)+F_{n-k}(z)} .
$$

Proof. We proceed by induction on $k$. The case $k=1$ is (2.1). Assume for $k>1$ and $n>k$ we have

$$
F_{n}(z)=\frac{F_{k}(z) F_{n-k}(z)-1}{F_{k}(z)+F_{n-k}(z)} .
$$

Now suppose $n>k+1$. Then $F_{n}$ satisfies (2.2). From (2.1) we obtain

$$
F_{k}(z)=\frac{z F_{k+1}(z)+1}{z-F_{k+1}(z)} .
$$

By employing the latter for $F_{k}$ and the definition of $F_{n-k}$ in $(2.2)$, the result follows by straightforward computations.

Lemma 2.2. For $n \geq 1$ and $k \geq 1, F_{n} \circ F_{k}=F_{n k}$.

Proof. We proceed by induction on $n$. The case $n=1$ and $k \geq 1$ follows from the definition of $F_{1}$. By employing (2.1), the induction hypothesis, and Lemma 2.1 , we have

$$
\begin{aligned}
F_{n+1}\left(F_{k}(z)\right) & =\frac{F_{k}(z) F_{n}\left(F_{k}(z)\right)-1}{F_{k}(z)+F_{n}\left(F_{k}(z)\right)} \\
& =\frac{F_{k}(z) F_{n k}(z)-1}{F_{k}(z)+F_{n k}(z)} \\
& =F_{n k+k}(z) .
\end{aligned}
$$

Corollary 2.3. For $n \geq 1$ and $k \geq 1, F_{n} \circ F_{k}=F_{k} \circ F_{n}$.

Lemma 2.4. For $n \geq 2$, degree of $F_{n}$ is at least 2.

Proof. Suppose $F_{n}(z)=\frac{p_{n}(z)}{q_{n}(z)}$ where $p_{n}$ and $q_{n}$ are two polynomials. It can be shown by induction that $p_{n}(z)=z p_{n-1}(z)-q_{n-1}(z)$ and $q_{n}(z)=z q_{n-1}(z)+$ $p_{n-1}(z)$ where $p_{1}(z)=z$ and $q_{1}(z)=1$. Since $\operatorname{deg} p_{1}>\operatorname{deg} q_{1}$ and $\operatorname{deg} p_{n}=$ $\operatorname{deg} p_{n-1}+1$, therefore $\operatorname{deg} p_{n}>\operatorname{deg} q_{n}$ and consequently $F_{n}(\infty)=\infty$. By 
induction on $n$ and employing (2.1) one can easily see that $\pm i$ are the fixed points of $F_{n}$. Thus $\operatorname{deg} F_{n} \geq 2$ unless $F_{n}(z)=z$ that is impossible.

The following theorem will determine the Julia set of $F_{n}$.

Theorem 2.5. For $n \geq 2$, the Julia set of $F_{n}$ is $\mathbb{R} \cup\{\infty\}$.

Proof. We first show that the Julia set of $F_{2}(z)=\frac{z^{2}-1}{2 z}$ is $\mathbb{R} \cup\{\infty\}$. The points $z= \pm i$ are attracting fixed points and $\infty$ is a repelling fixed point of $F_{2}$. Therefore $\infty$ belongs to $J\left(F_{2}\right)$ and the set of all iterated preimages of $\infty$, $\left\{z \in \hat{\mathbb{C}}: F_{2}^{n}(z)=\infty\right.$ for some $\left.n \geq 0\right\}$, is everywhere dense in $J\left(F_{2}\right)$ (see [4, Corollary 4.13]). Since all of the solutions of $\frac{z^{2}-1}{2 z}=c$ for real $c$ are real, we conclude that $J\left(F_{2}\right) \subseteq \mathbb{R} \cup\{\infty\}$. Since the boundary of the basins of attraction of the fixed points $i$ and $-i$ are subsets of $J\left(F_{2}\right)$, the upper half-plane is the basin of attraction of $i$ and the lower half-plane is the basin of attraction of $-i$. Therefore $J\left(F_{2}\right)=\mathbb{R} \cup\{\infty\}$.

On the other hand, by Corollary $2.3, F_{2} \circ F_{n}=F_{n} \circ F_{2}$. Thus $J\left(F_{n}\right)=$ $J\left(F_{2}\right)=\mathbb{R} \cup\{\infty\}$ (see [1, Theorem 4.2.9]).

By differentiating of (2.1) one can see that the fixed points $\pm i$ are attracting. By applying these fixed points we are going to prove the following theorem.

Theorem 2.6. $F_{n}$ and $z^{n}$ are conjugate.

Proof. Suppose $\varphi$ is a Möbius function such that $\varphi(0)=-i$ and $\varphi(\infty)=i$. Thus $\varphi(z)=i \frac{z-a}{z+a}$, for some $a \in \mathbb{C}$. By induction on $n$ and employing (2.1), we conclude that $F_{n} \circ \varphi(z)=i \frac{z^{n}+a^{2}}{z^{n}-a^{2}}$. It can be shown that $F_{n} \circ \varphi(z)=\varphi\left(z^{n}\right)$ if and only if $a=-1$. Thus $F_{n}$ and $z^{n}$ are conjugate under $\varphi(z)=i \frac{z+1}{z-1}$.

Remark 1 .

- In the proof of Theorem 2.6, it is possible to suppose $\varphi(0)=i$ and $\varphi(\infty)=-i$.

- Since the image of the unit circle under $\varphi$ in Theorem 2.6 is the extended real line, it is possible to conclude Theorem 2.5 from Theorem 2.6 .

Now we are going to describe $F_{n}(z)=\frac{p_{n}(z)}{q_{n}(z)}$ more specifically. By Theorem 2.6, $F_{n}$ is a rational function of degree $n$ and $p_{n}$ and $q_{n}$ are two relatively prime polynomials of degree $n$ and $n-1$, respectively. The following proposition shows that $F_{n}$ has $n$ distinct real roots and $n-1$ real poles.

Proposition 2.7. All the roots of $q_{n}$ and $p_{n}$ are real and distinct.

Proof. According to the above discussion, $F_{n}(z)=\infty$ if only if $q_{n}(z)=0$ or $z=\infty$. On the other hand $F_{n}(z)=\infty$ if and only if $\varphi\left(\left(\varphi^{-1}(z)\right)^{n}\right)=\infty$. Since $\varphi(1)=\infty$, therefore $\left(\varphi^{-1}(z)\right)^{n}=1$. Thus $\varphi^{-1}(z)$ must be an $n$-th root of unity (i.e., $\left.1, e^{\frac{2 \pi i}{n}}, \ldots, e^{\frac{2(n-1) \pi i}{n}}\right)$ and consequently $z=\infty, \varphi\left(e^{\frac{2 \pi i}{n}}\right), \ldots, \varphi\left(e^{\frac{2(n-1) \pi i}{n}}\right)$. Therefore $q_{n}(z)=0$ has $n-1$ distinct roots that are real. 

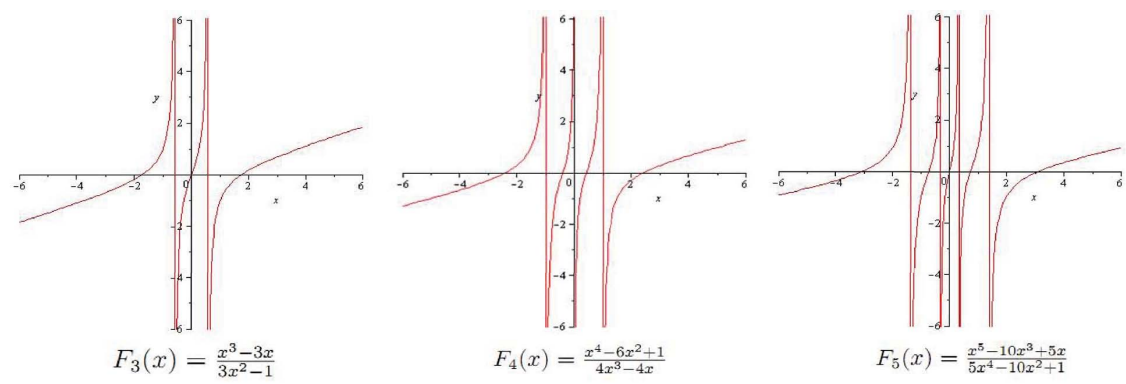

FiguRE 1. The graph of $F_{n}$, for $n=3,4,5$

In addition, $p_{n}(z)=0$ if and only if $F_{n}(z)=0$. Since $\varphi(-1)=0$, the similar argument shows that $F_{n}(z)=0$ if and only if $\left(\varphi^{-1}(z)\right)^{n}=-1$. Thus $p_{n}$ has $n$ real and distinct roots.

Figure 1 illustrates the roots and poles of $F_{n}(z)$ for $n=3,4,5$.

\section{3. $F_{n}$ as a real rational function}

In this section we consider $F_{n}$ as a real function and show that $F_{n}$ is chaotic on some subset of $\mathbb{R}$. Figure 1 shows the graph of $F_{n}$, for $n=3,4,5$.

Since $F_{n}$ is not defined on the roots of $q_{n}$ as a real function, we are only able to study the dynamics of $F_{n}$ on the set $\mathbb{R} \backslash B_{n}$, where

$$
B_{n}=\bigcup_{k=0}^{\infty} F_{n}^{-k}\left(\left\{x \in \mathbb{R}: \quad x \text { is a pole of } F_{n}\right\}\right)=\bigcup_{k=1}^{\infty} F_{n}^{-k}(\infty)
$$

Definition of $B_{n}$ shows that $F_{n}\left(\mathbb{R} \backslash B_{n}\right) \subseteq \mathbb{R} \backslash B_{n}$.

On the other hand $\varphi\left(e^{i \theta}\right)=i \frac{e^{i \theta}+1}{e^{i \theta}-1}=\cot \frac{\theta}{2}$. Thus if $C(\theta)=\cot \frac{\theta}{2}$ and $D_{n}: S^{1} \rightarrow S^{1}$ is given by

$$
D_{n}(\theta)=n \theta \quad(\bmod 2 \pi),
$$

where $S^{1}$ is the unit circle, then the relation $F_{n} \circ \varphi(z)=\varphi\left(z^{n}\right)$ can be written as $F_{n} \circ C(\theta)=C \circ D_{n}(\theta)$ when $z=e^{i \theta}$. Therefore if we reduce the domain of $D_{n}$ to $S^{1} \backslash A_{n}$, where $A_{n}=\cup_{k \geq 1} D_{n}^{-k}(0)$, then the diagram

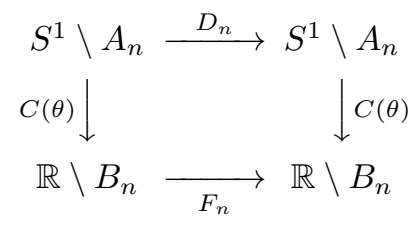

is commutative. Since $A_{n}$ is dense in $S^{1}, C\left(A_{n} \backslash\{0\}\right)=B_{n}$, and $C(\theta)$ is a homeomorphism, if $0<\theta<2 \pi$, then we have the following result.

Proposition 3.1. $B_{n}$ is dense in $\mathbb{R}$. 
It is known that $D_{n}: S^{1} \rightarrow S^{1}$ is chaotic (see [2, page 50]). Now we will show that

Theorem 3.2. $F_{n}$ is chaotic on $\mathbb{R} \backslash B_{n}$.

Proof. First we will show that $D_{n}: S^{1} \backslash A_{n} \rightarrow S^{1} \backslash A_{n}$ is chaotic. Note that

(1) The periodic points of $D_{n}$ are dense in $S^{1}$. The points of $A_{n}$ are eventually maps to 0 , so $x \neq 0$ and $x \in A_{n}$ is not a periodic point of $D_{n}$. Thus the periodic points of $D_{n}$ are dense in $S^{1} \backslash A_{n}$.

(2) For the nonempty open subset $U$ of $S^{1}$, there is $k \in \mathbb{N}$ such that $D_{n}^{k}(U)=S^{1}$. Therefore $D_{n}^{k}\left(U \backslash A_{n}\right)=S^{1} \backslash A_{n}$ since $D_{n}\left(A_{n}\right)=A_{n}$. If $V$ is a nonempty open subset of $S^{1}$, then $D_{n}^{k}\left(U \backslash A_{n}\right) \cap\left(V \backslash A_{n}\right) \neq \emptyset$. This implies that $D_{n}$ is transitive on $S^{1} \backslash A_{n}$.

(3) Since $D_{n}^{k}\left(U \backslash A_{n}\right)=S^{1} \backslash A_{n}$ for some $k \in \mathbb{N}$, if $x \in U \backslash A_{n}$, then there is $y \in U \backslash A_{n}$ such that $d\left(D_{n}^{k}(x), D_{n}^{k}(y)\right)>1 / 2$. Thus $D_{n}$ has sensitive dependence on initial conditions on $S^{1} \backslash A_{n}$.

Therefore $D_{n}$ is chaotic on $S^{1} \backslash A_{n}$.

Since the diagram (3.1) is commutative, the density of the periodic points of $F_{n}$ and topological transitivity of $F_{n}$ are concluded easily from the above discussion. Also for each open set $U$ in $\mathbb{R} \backslash B_{n}$ there is $k \in \mathbb{N}$ such that $F_{n}^{k}(U)=\mathbb{R} \backslash B_{n}$. Thus $F_{n}$ has sensitive dependence on initial conditions on $\mathbb{R} \backslash B_{n}$.

Acknowledgements. The authors would like to thank Professor Amir Akbary for some editorial corrections.

\section{References}

[1] A. F. Beardon, Iteration of Rational Functions, Cambridge, 1991.

[2] R. Devaney, An Introduction to Chaotic Dynamical Systems, 2nd. ed., Addison-Wesley, 1989.

[3] J. Milnor, Pasting together Julia sets: A worked out example of mating, Experiment. Math. 13 (2004), no. 1, 55-92.

[4] _ Dynamics in One Complex Variable, 3rd. ed., Princeton University Press, 2006.

MONIREH AKBARI

Shahid Rajaee Teacher Training University

TEHran, 16788-15811, IRAN

E-mail address: akbari@srttu.edu

Maryam RaBiI

AlzAhra University

TEhran, 19938-93973, IrAN

E-mail address: mrabii@alzahra.ac.ir 